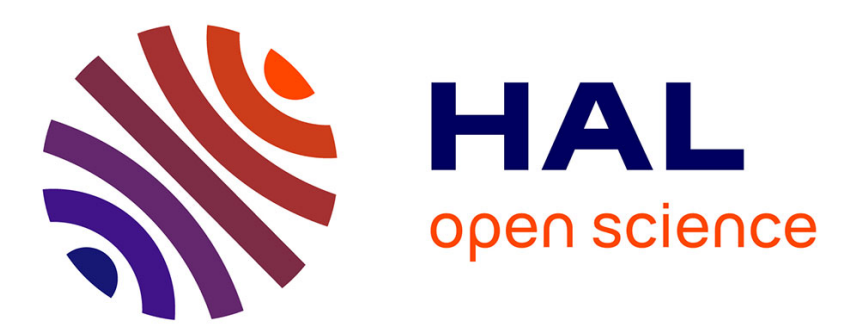

\title{
Thresholds and distances to better detect wet snow over mountains with Sentinel-1 image time series
}

Fatima Karbou, Guillaume James, Philippe Durand, Abdourrahmane Atto

\section{To cite this version:}

Fatima Karbou, Guillaume James, Philippe Durand, Abdourrahmane Atto. Thresholds and distances to better detect wet snow over mountains with Sentinel-1 image time series. Change Detection and Image Time Series Analysis, 1: Unsupervised Methods, Wiley, pp.1-18, 2021, 10.1002/9781119882268.ch5 . hal-03483209

\section{HAL Id: hal-03483209 \\ https://hal.inria.fr/hal-03483209}

Submitted on 16 Dec 2021

HAL is a multi-disciplinary open access archive for the deposit and dissemination of scientific research documents, whether they are published or not. The documents may come from teaching and research institutions in France or abroad, or from public or private research centers.
L'archive ouverte pluridisciplinaire HAL, est destinée au dépôt et à la diffusion de documents scientifiques de niveau recherche, publiés ou non, émanant des établissements d'enseignement et de recherche français ou étrangers, des laboratoires publics ou privés. 


\title{
THRESHOLDS AND DISTANCES TO BETTER DETECT WET SNOW OVER MOUNTAINS WITH SENTINEL-1 IMAGE TIME SERIES
}

\author{
Fatima KARBOU ${ }^{1}$, Guillaume JAMES ${ }^{2}{ }^{3}$, Philippe DURAND ${ }^{4}$, Abdourrahmane \\ ATTO $^{5}$ \\ 1 Univ. Grenoble Alpes, Université de Toulouse, Météo-France, CNRS, CNRM, \\ Centre d'Études de la Neige, Grenoble, France ${ }^{2}$ INRIA Grenoble - Rhône- \\ Alpes, Tripop Team $\quad{ }_{3}^{3}$ Univ. Grenoble Alpes, CNRS, Inria, Grenoble INP*, LJK, \\ 38000 Grenoble, France. * Institute of Engineering Univ. Grenoble Alpes $\quad{ }^{4}$ CNES, \\ Toulouse, France. $\quad{ }^{5}$ LISTIC, Université Savoie Mont Blanc, Annecy, France
}

\subsection{Introduction}

In mountain regions, seasonal snow monitoring is very important for many applications such as hydrology, mountain ecosystems, meteorology and avalanche forecasting. For example, a good knowledge of snow extent, of its evolution over time and in particular the starting date of snow-melt is of prime importance for hydro power production and for anticipating flood risks. A good knowledge of starting date of snow-melt is also of great importance for mountain ecosystem studies. Space-based remote sensing allows monitoring of seasonal snow at scales of time and space that are not comparable to ground-based measuring stations. The 
Sentinel-1 satellites provide cloud insensitive C-band Synthetic Aperture Radar (SAR) data and allow snow cover to be observed day and night at unprecedented temporal and spatial resolutions. The sensitivity of the measurements to snow liquid water content has enabled the development and evaluation of algorithms for detecting wet snow (Nagler et al. (2016), Baghdadi \& Bernier (1998), Magagi \& Bernier (2003), Karbou et al. (2021)). SAR observations, however, require heavy pre-processing steps and are complex to use in areas of high relief. Most wet snow detection algorithms are based on the use of thresholds to distinguish wet snow from dry snow or soil by comparing a winter image with a reference image without snow. This approach gives satisfactory results during melting periods, but has important limitations. In the literature, there are several studies on this topic. Nagler \& Rott (2000) used a fixed threshold to map wet snow using a reference image taken under either dry snow or frozen ground conditions. However, the use of a threshold of 2-3 $\mathrm{dB}$ does not always guarantee good results because detection errors may occur in situations linked to some specific surface types, snow surface roughness values, moisture content (Baghdadi et al. (2000)). The use of a fixed threshold is particularly problematic in forest areas where the variation of the SAR signal is relatively small (sometimes below the 2-3 dB range). The interaction of the SAR signal and the forest depends on the density and structure of the vegetation. The seasonal phenology of trees with pre-winter leaf fall can also have a significant impact on radar signals. To date, few studies have focused on the detection of wet snow in forested areas. Koskinen et al. (1997) suggested using two reference images, instead of one, taken before and after the melting season to better estimate the relative fraction of snow-free soil. This does not solve all problems as false detection of wet snow occur if the soil in the second reference image was already drying (Luojus et al. (2007)). Marin et al. (2020) have recently shown that the melting phases (moistening, ripening and runoff) can be identified using multi-temporal SAR backscatter. Other studies have used threshold functions instead of a fixed threshold to better account for signal variability with land cover (e.g. sigmoid functions have been used by Malnes \& Guneriussen (2002), Longépé et al. 2009, Rondeau-Genesse et al. 2016). Optical satellite measurements have also been used to correct possible inconsistencies in wet snow estimates, particularly in forests or waterlogged soils (Rondeau-Genesse et al. 2016). In this chapter we discuss wet snow extent estimation using Nagler's backscatter thresholding method (noted reference method here after). We also examine some other interesting options to improve wet snow detection by exploring the use of similarity metrics (such as the normalized cross correlation ratio, the Hausdorff distance and some other more refined image distance functions). Several distances are calculated and tested on a database of Sentinel-1 SAR image time series of different sizes over the French Alps. The resulting metrics are discussed and compared with the reference method as well as with independent data. 


\subsection{Test area and data}

We use as observations the C-band backscatter coefficients from the SAR instrument aboard the European Space Agency (ESA) Sentinel-1 satellites as part of the Copernicus program. The Sentinel-1 missions were launched in April 2014 and April 2016 respectively. On our test areas, the revisit period is 6 days. We are using level 1 products from the Groud Range Detected (GRD, with a spatial resolution of $20 \mathrm{~m}$ in VV and VH polarizations) available on the CNES and ESA websites (https://scihub.copernicus.eu/dhus/; https://peps.cnes.fr). For each orbit direction (ascending, descending), we use one image every 6 days between August 1, 2017 and August 31,2018, resulting in a continuous time series of more than 130 images. The time period of this study includes a highly disturbed meteorological and snow winter conditions (Goetz (2018), Stoffel \& Corona (2018)). We rely on CNES facilities to perform pre-processing of SAR images using the Orfeo ToolBox software. The pre-processing thermal noise removal, speckle filtering, radiometric calibration and terrain correction using the SRTM Digital Elevation Model (DEM) at $30 \mathrm{~m}$. The DEM is also used to link each pixel of the satellite images with an altitude, slope and orientation. For evaluation and comparison purposes, we make use of in-situ snow measurements from the Météo-France station network and we also use Theia snow products derived from cloud free optical Sentinel-2 satellite date (Gascoin et al. $($ n.d.)). Our test zones are heterogeneous steep mountain areas located in the French Alps with a complex topography and a wide variety of surface types; we have chosen three embedded zones of different areas as shown in Figure 5.1. Area1 with 10900x10900 pixels, Area2 with 1759x1381 pixels and Area3 with 24x28 pixels.

\subsection{On wet snow detection using Sentinel-1}

Change detection is a feature of interest for snow monitoring in the mountains because it allows to identify and analyse changes in a scene from images acquired at different dates. Radar imagery has the ability to observe at any time of the day or night, and is therefore very useful in complex situations with poor weather conditions. The challenge is to isolate the changes occurring over a time series of images and to accurately assign these changes in the image to some environment changes (related to the state of snow, vegetation, soil, ...). Unsurprisingly, change detection is widely used for wet snow detection from SAR images (Nagler et al. (2016), Baghdadi \& Bernier (1998), Magagi \& Bernier (2003)). A review of methods for wet snow detection using SAR data is given in Tsai et al. (2019). C-band measurements are sensitive to wet snow due to the high dielectric contrast with non wet snow covered surfaces, as dry snow is generally transparent at C-band. An image ratio (with and without snow) is calculated and a threshold is applied to obtain a wet snow mask.

Figure 5.2 shows Altitude-time diagrams of VH SAR backscatters from the Sentinel-1 ascending orbit A161 (early morning) computed for test area2 and for 


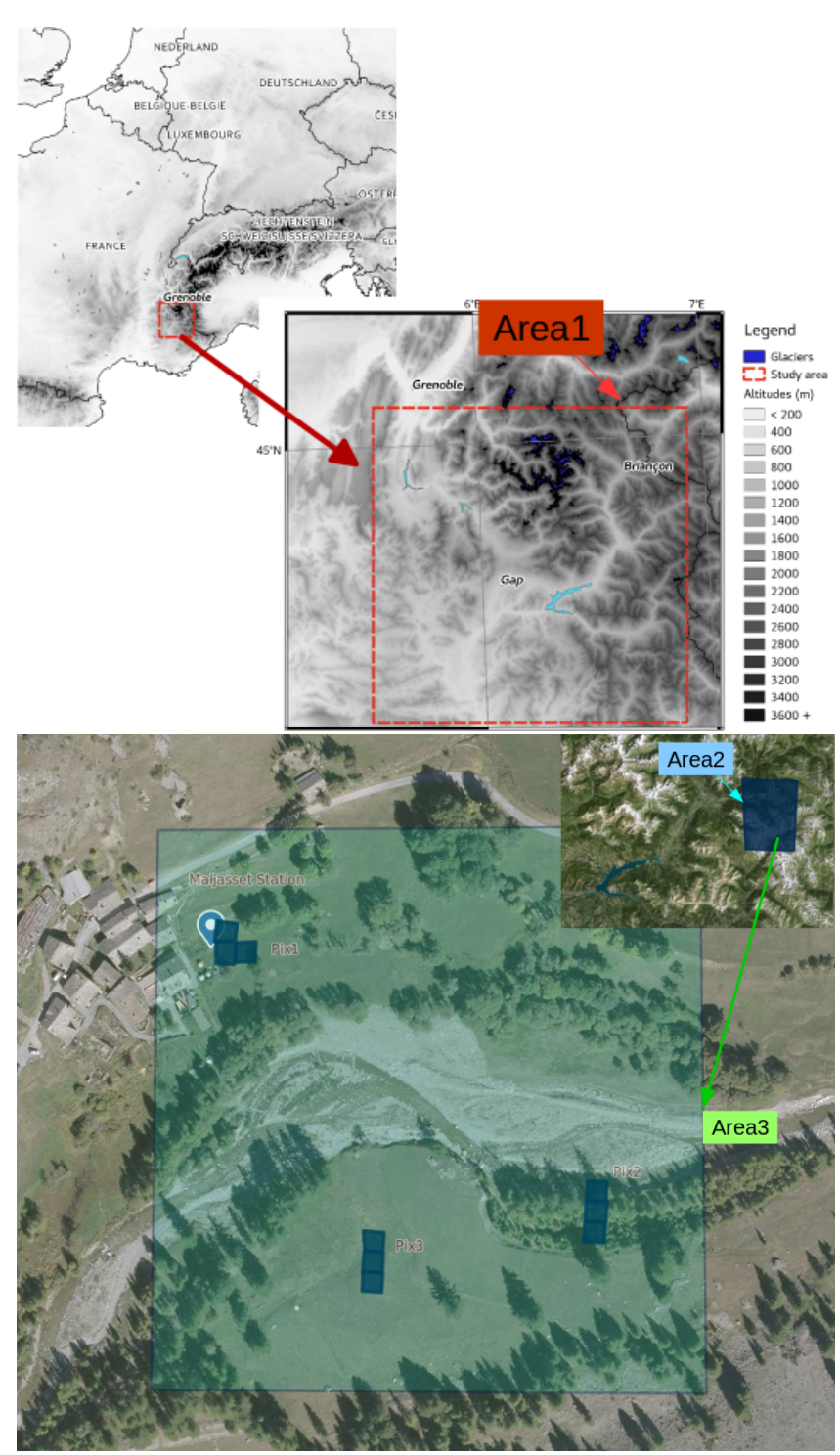

Figure 5.1: Location of the study areas.

southern orientations. Altitudes vary between $1200 \mathrm{~m}$ and $3100 \mathrm{~m}$ and mean backscatters range from $-20 \mathrm{~dB}$ to $-8 \mathrm{~dB}$. We observe signal variations throughout the season that may be partly due to rain-on-snow effects and snowmelt (from the end of March 2018). This is characterised by a significant drop in the backscatter for some 


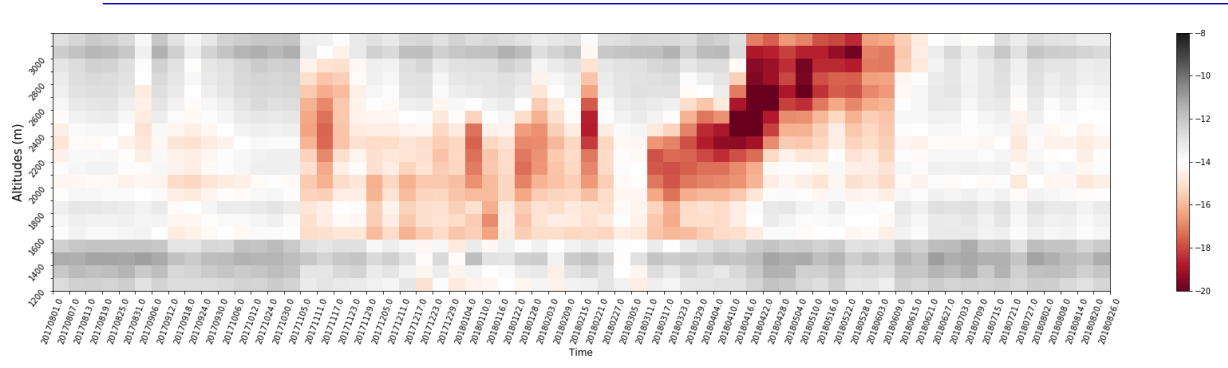

(a)

Figure 5.2: Altitude-time diagrams of VH SAR backscatters from the ascending orbit A161 (early morning) computed for test area2 and for southern orientations

individual dates associated with rain-on-snow events such as early January 2018, 22nd of January 2018, mid of February and mid of March 2018... We also observe a continuous decrease of the bakcscatter starting at the end of March coinciding with the beginning of the snowpack melting.

In order to look more specifically at the variation of the SAR signal during the 2017-2018 season, we selected a small alpine area at about $1900 \mathrm{~m}$ close to the insitu station Maljasset (called Maljasset) from the Météo-France station network from which daily measurements of snow depth, rain rate as well as temperature (min, max) are made. Within the test area3 (shown in Figure 5.1), 3 small sites are selected in the vicinity of the Maljasset station: an open area very close to the station (called Pix1-NearStation), a wooded zone (called Pix2-Forest), and another open area (called Pix3-Grassland). For each of these selected areas three Sentinel-1 observation pixels were monitored over one year (August 2017 to July 2018, pixels shown in blue squares in Figure 5.1.

Figure 5.3 shows time series of Sentinel-1 VH observations from the ascending orbit for our 3 selected sites in Area3 (see location in Figure 5.1). Results are shown for Pix1-NearStation (black curves), Pix2-Forest (green curves), and for Pix3-Grassland (magenta curves). Daily snow heights and rain rates measured at the Maljasset station are displayed as shaded blue areas and green bars respectively. We note that, overall, wooded areas have higher backscatter coefficients than open or mixed areas. We also notice fluctuations in the SAR signal, which varies depending on the type of surface, some of these fluctuations can be explained by the effects of snow accumulation or rain on snow. One could also notice an overall gradual increase of backscatters with snow accumulation until starting of melting process; the increase in backscattering may be due to snow volume scattering; this effect is more pronounced for morning passes and open areas (the increase of backscatters with snow accumulation is also observed for VV polarisation (not shown)). Regarding the evolution of temperatures 


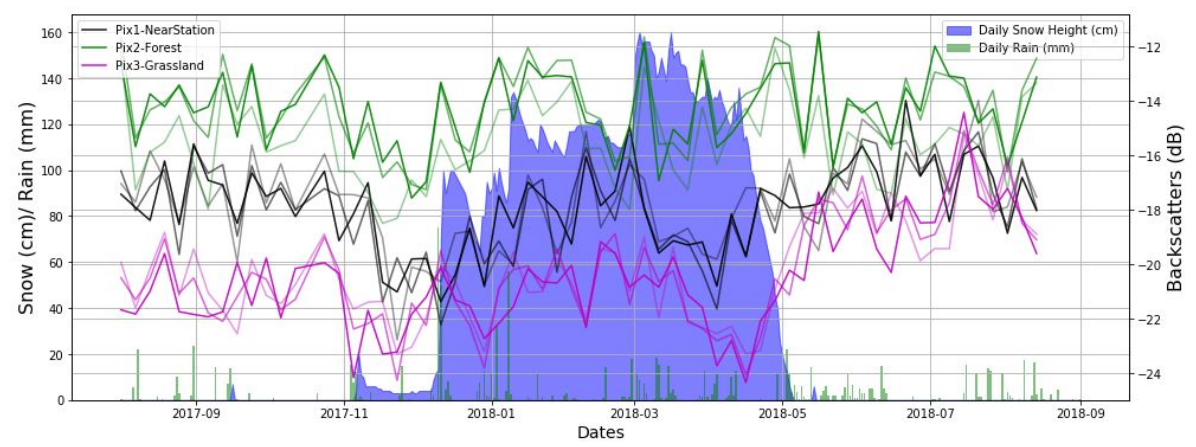

(a)

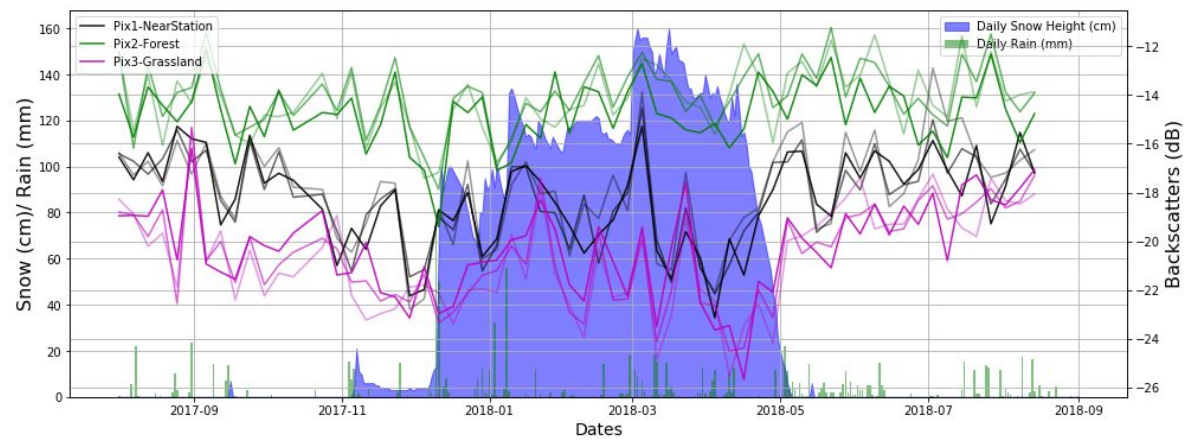

(b)

Figure 5.3: (a) Time series of Sentinel-1 VH observations from the ascending orbit for 3 selected sites in the vicinity of the measuring station Maljasset (see its location in Fig1). Results are shown for a mixed zone very close to the station (called Pix1NearStation, curves in black), a wooded zone (called Pix1-Forest, curves in green), and an open area (grassland) zone (called Pix1-Grassland, curves in magenta). For each of these selected areas three observation pixels were monitored over time (shown in blue in Figure 5.1]. For each of these areas three observation pixels were monitored over time. Daily snow heights measured at the Maljasset station are displayed as shaded blue areas and green bars indicate measured daily rainfall rates (in $\mathrm{mm}$ ). (b) same as (a) but for descending orbit.

(not shown), we note roughly three main periods of variation: August-October 2017 with summer conditions, Nov-mid April with much lower temperatures (the minimum reaching $-23^{\circ}$ at the end of February) and a third period until August 2018 with again more moderate temperatures. Marin et al. (2020) proposed simple rules for identifying snow melting phases through the analysis of SAR time series. A decrease in the afternoon backscatters (i.e., with the ascending orbit) of at least $2 \mathrm{~dB}$ or more from 
THRESHOLDS AND DISTANCES TO BETTER DETECT WET SNOW OVER MOUNTAINS WITH SENTINEL-1 IMAGE TIME SERIES 7

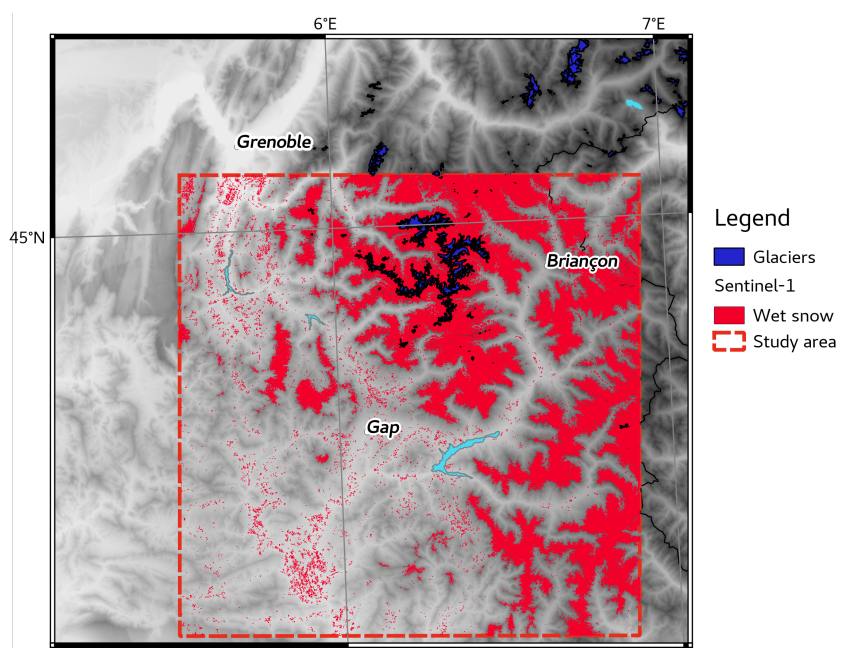

(a)

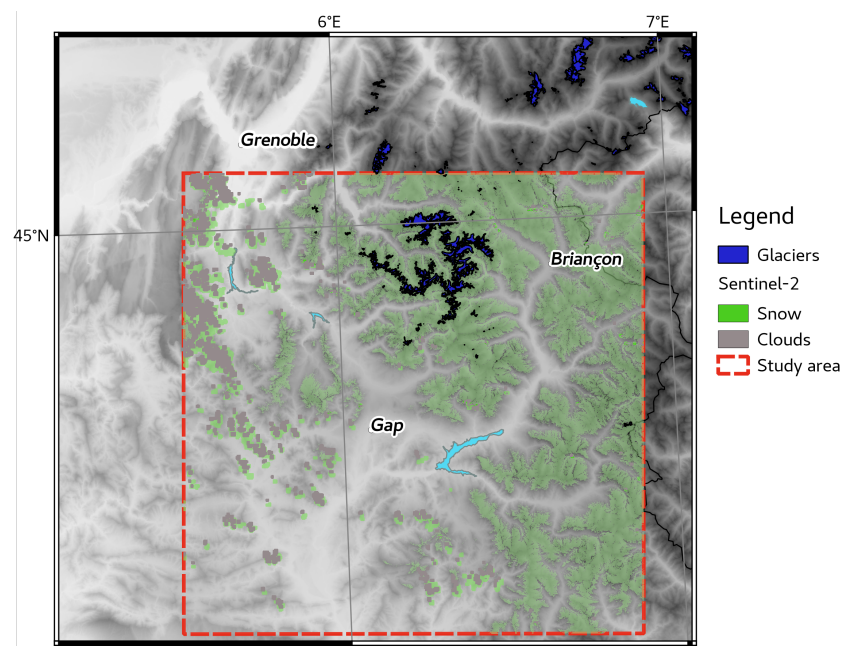

(b)

Figure 5.4: (a) Wet snow cover extent derived from Sentinel-1 descending/ascending images of 27/28 April 2018 and (b) snow extent masq derived from Sentinel-2 optical image of 25th April 2018. Background: SRTM Digital elevations.

the winter trend would indicate the beginning of the melting process. The ripening phase is identified as soon as this phenomenon is also observed in the morning time series (descending orbit). This phase, characterized by several oscillations, ends when 
the morning and afternoon backscatters reach local minimums. The authors suggest taking the mean date among the local minima as the beginning of the runoff phase, which is characterized by a monotonous increase in the backscatter coefficient. It is particularly difficult to apply these rules over our study period, as the winter of 2017-2018 was exceptionally eventful with successive weather events bringing a lot of snow and rain on snow (rain on snow events mid December, early January, ....). The snow depth and rainfall rate curves measured at Maljasset station indicate the extent of these events. Following Marin et al. (2020) rules and apart from rain-on-snow events, one could notice a strong decrease of afternoon backscatters on March 22-23, 2018. This decrease is much more pronounced for mixed and open areas than for areas with trees (over which less than $2 \mathrm{~dB}$ of change is noticed). Prior to this, several signal oscillations are observed and there is a continuous decrease in backscatter (evening) until April 16 for mixed areas and later for unvegetated areas (April 28) before backscatters begin to increase. Signals over forested areas are more complex to interpret despite some variations due to snow or rain-on-snow events.

Another example at larger scale is given in Figure 5.4 (over test Area1). The figure compares (a) wet snow detection derived from Sentinel-1 images of 27-28 April 2018 from descending and ascending orbits using a 2dB threshold method (late August 2017 being used as reference) with (b) snow extent obtained from Sentinel-2 optical image of 25th April 2018. Sentinel-2 scene was almost cloud-free and the agreement between Sentinel-1 and Sentinel-2 snow products is rather good for this period. It should be noted that Sentinel-2 measurements detect the extent of total snow, whether dry or wet, while Sentinel-1 detects only wet snow, so the comparison is not between two identical products. In addition, observations from the two satellites are not acquired at identical time-of-day.

\subsection{Metrics to detect wet snow}

As discussed in the introduction, there are several possible limitations to the use of threshold methods. For instance, there is the need to optimize the selection of one or two reference images as this is a key factor in wet snow detection results. There is also the need to adjust the threshold values to better account for the variability of the SAR signal by surface type. To discuss these issues in further detail, we propose to explore the potential of different mathematical metrics that would (1) allow for better detection of snow cover induced changes in SAR image time series by maximizing the sensitivity to wet snow, and/or (2) make a better selection of reference images. In this chapter, we discuss the usefulness of robust image comparison metrics to isolate wet snow pixels (see Zamperoni \& Starovoitov (1996), Di Gesu \& Starovoitov (1999), Starovoitov \& Samal (1998) and references therein for reviews on distance-based image comparison in a more general context). We test different similarity measures based on the normalized cross correlation ratio, different matrix norms and the Hausdorff distance. 
THRESHOLDS AND DISTANCES TO BETTER DETECT WET SNOW OVER MOUNTAINS WITH SENTINEL-1 IMAGE TIME SERIES 9

In order to define different distances, we start by introducing some matrix norms. Given a real $m \times n$ matrix $A$ and $p \in[1, \infty]$, we define the norms

$$
\begin{aligned}
& \text { for } p \in[1, \infty),\|A\|_{p}=\left(\sum_{1 \leqslant i \leqslant m, 1 \leqslant j \leqslant n}\left|a_{i j}\right|^{p}\right)^{1 / p}, \\
& \|A\|_{\infty}=\max _{1 \leqslant i \leqslant m, 1 \leqslant j \leqslant n}\left|a_{i j}\right| .
\end{aligned}
$$

Classical values correspond to $p=1$ (mean error), $p=2$ (root-mean-squared or rms error) and $p=\infty$ (maximal error, or supremum norm), the latter being more sensitive to the perturbation of a small number of matrix coefficients for large matrices. The norms (5.1) apply in particular to vectors (case $m=1$ or $n=1$ ). We also consider the operator norms (or induced norms) defined by

$$
\|A\|_{p}=\sup _{x \in \mathbb{R}^{n}, x \neq 0} \frac{\|A x\|_{p}}{\|x\|_{p}}
$$

our main focus being $p=2$ (spectral norm). It is a classical result that $\|A\|_{2}$ is the largest singular value of $A$, i.e. the square root of the largest eigenvalue of $A^{T} A$, where $(\cdot)^{T}$ denotes the transpose. Each of the above matrix norms \|\| induces a distance $D(A, B)=\|A-B\|$.

Another similarity measure between two $m \times n$ nonzero matrices $A, B$ is given by the normalized cross correlation ratio

$$
\mathrm{CO}(A, B)=1-\frac{1}{\|A\|_{2}\|B\|_{2}} \sum_{1 \leqslant i \leqslant m, 1 \leqslant j \leqslant n} a_{i j} b_{i j}
$$

which vanishes if and only if $B=\lambda A$ for some positive constant $\lambda$.

The Hausdorff distance provides another similarity measure which can be interesting to capture small interface motions within images, while being more sensitive than \|\|$_{1}$ and \|\|$_{2}$ to localized perturbations. The Hausdorff distance $\mathrm{H}$ between two real $m \times n$ matrices $A, B$ is defined as follows. Each matrix is identified with a function defined on a grid of size $m \times n$, and one considers the Hausdorff distance between the two corresponding graphs

$$
\begin{aligned}
G_{A} & =\left\{A_{i j}=\left(\frac{i}{m}, \frac{j}{n}, a_{i j}\right), 1 \leqslant i \leqslant m, 1 \leqslant j \leqslant n\right\}, \\
G_{B} & =\left\{B_{k l}=\left(\frac{k}{m}, \frac{l}{n}, b_{k l}\right), 1 \leqslant k \leqslant m, 1 \leqslant l \leqslant n\right\} .
\end{aligned}
$$


In this work, we measure the distance between points with the city block distance \|\|$_{1}$, leading to $\left\|A_{i j}-B_{k l}\right\|_{1}=\frac{|i-k|}{m}+\frac{|j-l|}{n}+\left|a_{i j}-b_{k l}\right|$. We have then

$$
\mathrm{H}(A, B)=\max _{1 \leqslant i \leqslant m, 1 \leqslant j \leqslant n}\left\{\mathrm{~d}\left(A_{i j}, G_{B}\right), \mathrm{d}\left(B_{i j}, G_{A}\right)\right\},
$$

where $\mathrm{d}\left(A_{i j}, G_{B}\right)=\min _{B_{k l}}\left\|A_{i j}-B_{k l}\right\|_{1}$ and $\mathrm{d}\left(B_{i j}, G_{A}\right)$ is defined similarly.

It should be noted that the above distances lead to different computational costs. If $m \approx n$, the computation of the entrywise norms (5.1) and correlation (5.3) require $O\left(n^{2}\right)$ floating-point operations. Evaluating the spectral norm takes $O\left(n^{3}\right)$ operations and a naive algorithm for computing the Hausdorff distance requires $O\left(n^{4}\right)$ operations.

In this work, we use the following logarithmic similarity measures between nonnegative $m \times n$ matrices, which are based on the previous ones (5.1), (5.2), (5.3) and (5.4) :

$$
\begin{aligned}
& \mathrm{d}_{p}(A, B)=\|\ln A-\ln B\|_{p}, \quad p=1,2, \infty, \\
& \widetilde{\mathrm{d}}_{2}(A, B)=\|\ln A-\ln B\|_{2}, \\
& \mathrm{H}_{\log }(A, B)=\mathrm{H}(\ln A, \ln B), \\
& \mathrm{CO}_{\log }(A, B)=\mathrm{CO}(\ln A, \ln B),
\end{aligned}
$$

with the natural logarithm applied component-wise to the positive entry pairs :

$$
\begin{aligned}
& (\ln A)_{i j}= \begin{cases}\ln a_{i j} & \text { if } a_{i j}>0 \text { and } b_{i j}>0, \\
0 & \text { otherwise, }\end{cases} \\
& (\ln B)_{i j}= \begin{cases}\ln b_{i j} & \text { if } a_{i j}>0 \text { and } b_{i j}>0, \\
0 & \text { otherwise. }\end{cases}
\end{aligned}
$$

The sign condition allows one to neglect non-meaningful zero entries in the comparison of matrices arising from SAR images.

Moreover, when $B$ corresponds to a reference state with higher values of the matrix coefficients (absence of wet snow), the sensitivity to the decrease of coefficients in $A$ (presence of wet snow) can be enhanced by modifying the above distances in a suitable 
way. More precisely, we consider solely the entry pairs with $a_{i j} \leqslant b_{i j}$, which leads to the modified similarity measures :

$$
\begin{aligned}
& \mathrm{d}_{p}^{+}(A, B)=\left\|\ln A_{+}-\ln B_{+}\right\|_{p}, \quad p=1,2, \infty, \\
& \tilde{\mathrm{d}}_{2}^{+}(A, B)=\left\|\ln A_{+}-\ln B_{+}\right\|_{2}, \\
& \mathrm{H}_{\log }^{+}(A, B)=\mathrm{H}\left(\ln A_{+}, \ln B_{+}\right), \\
& \mathrm{CO}_{\log }^{+}(A, B)=\mathrm{CO}\left(\ln A_{+}, \ln B_{+}\right),
\end{aligned}
$$

where

$$
\left(\ln A_{+}\right)_{i j}=\left\{\begin{array}{ll}
\ln a_{i j} & \text { if } b_{i j} \geqslant a_{i j}>0, \\
0 & \text { otherwise }
\end{array} \quad\left(\ln B_{+}\right)_{i j}= \begin{cases}\ln b_{i j} & \text { if } b_{i j} \geqslant a_{i j}>0 \\
0 & \text { otherwise. }\end{cases}\right.
$$

It should be noted that these similarity measures are not symmetric with respect to the arguments $A$ (current image) and $B$ (reference image).

We will also consider modified distances restricted to a certain subset of indices $(i, j)$, corresponding for example to a given range of altitudes. Let us consider a grid subset $\Gamma \subset\{1,2, \ldots, m\} \times\{1,2, \ldots, n\}$ (corresponding to a subset of pixels), a $m \times n$ matrix $A$, and define the $m \times n$ matrix $A_{\mid \Gamma}$ through

$$
\left(A_{\mid \Gamma}\right)_{i j}= \begin{cases}a_{i j} & \text { if }(i, j) \in \Gamma \\ 0 & \text { otherwise }\end{cases}
$$

For all similarity measures $D$ defined above in equations $5.5-(5.12)$, we denote

$$
D_{\mid \Gamma}(A, B)=D\left(A_{\mid \Gamma}, B_{\mid \Gamma}\right)
$$

Hereafter, we denote by "norme1", "rms" and "normeinf" the distances (5.5) or 5.9) with $p=1, p=2$ and $p=\infty$, respectively. In the same way, "normeop2" refers to 5.6 and (5.10), "haus" to 5.7) and (5.11, and "correl" to (5.8) and 5.12).

\subsection{Discussion}

Let us first look at the correlation between images. Here we consider the descending orbit SAR images on Area2. Figure 5.5 shows "1 minus correlation" matrix computed using SAR time series. As expected, correlations between images 


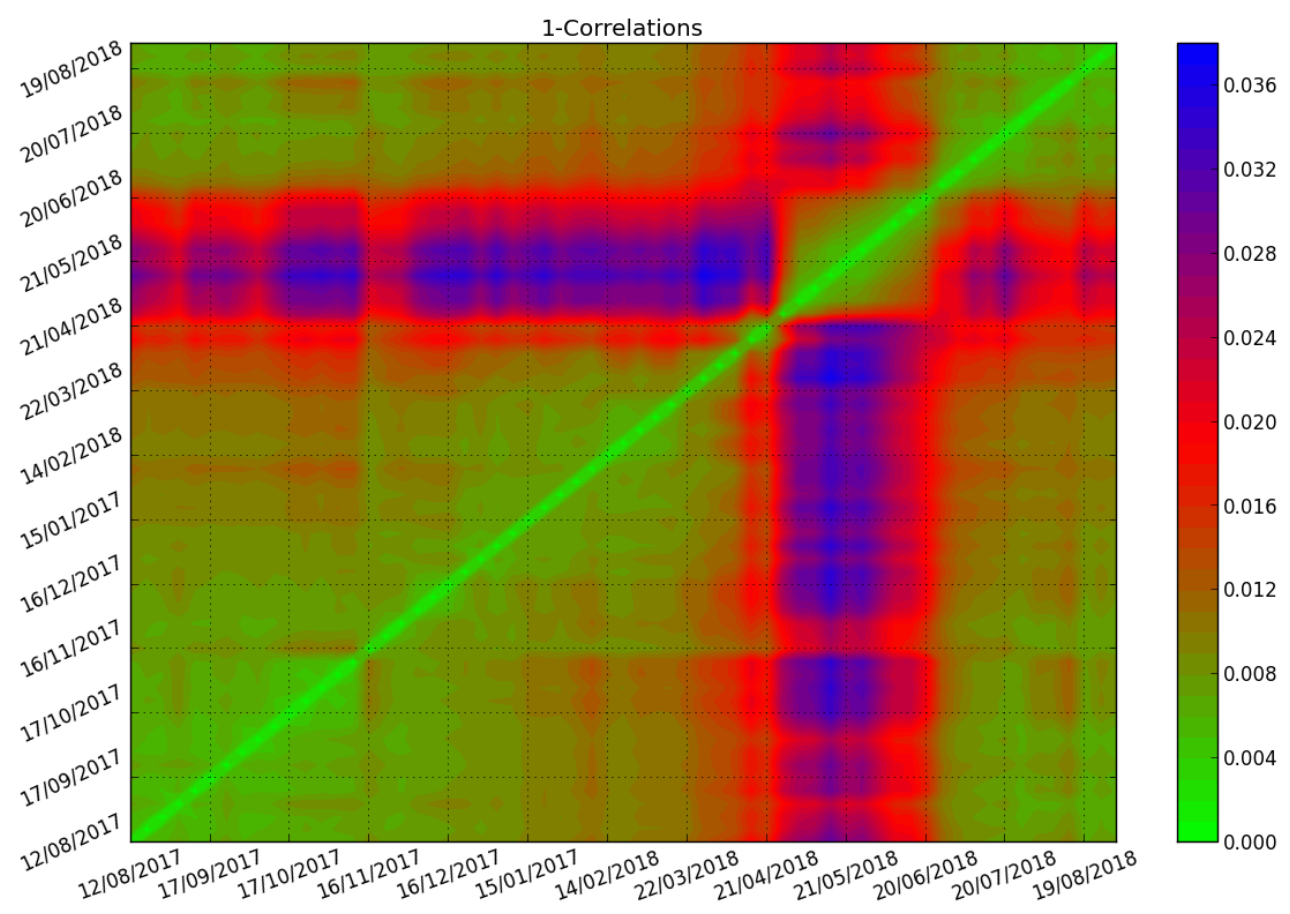

Figure 5.5: Correlation matrix of observation time series computed from formula (5.8).

decrease during the snowmelt period (roughly between 22/03/2018 and 20/06/2018). Outside the melting period, we find situations where the correlation decreases; this may be related to rain-on-snow events that significantly impact the observations. It is worth noting that the correlation matrix allows a global view of the entire time series and potentially better selection of one or more reference dates without running the risk of selecting a date already subject to significant fluctuations in the SAR signal. For instance, it would appear relevant to consider the image of October 17 as a reference rather than that of the end of August 2017.

Figure 5.6 shows the similarity curves obtained using Area3 SAR images (descending orbit) according to the following metrics defined in Section 5.4. correlation $\mathrm{CO}_{\log }$, mean error $\mathrm{d}_{1}$, maximal error $\mathrm{d}_{\infty}$, spectral norm $\tilde{\mathrm{d}}_{2}$ and Hausdorff distance $\mathrm{H}_{\mathrm{log}}$; the reference image is October 17th 2017. The distances are calculated on the area3 (see Figure 5.1 which includes the Maljasset station, whose measurements of snow depth and daily rain rate are also shown for comparison. The distance curves have been smoothed using the Legendre polynomial method for better readability (dotted symbols represent true estimates and solid lines are filtered 


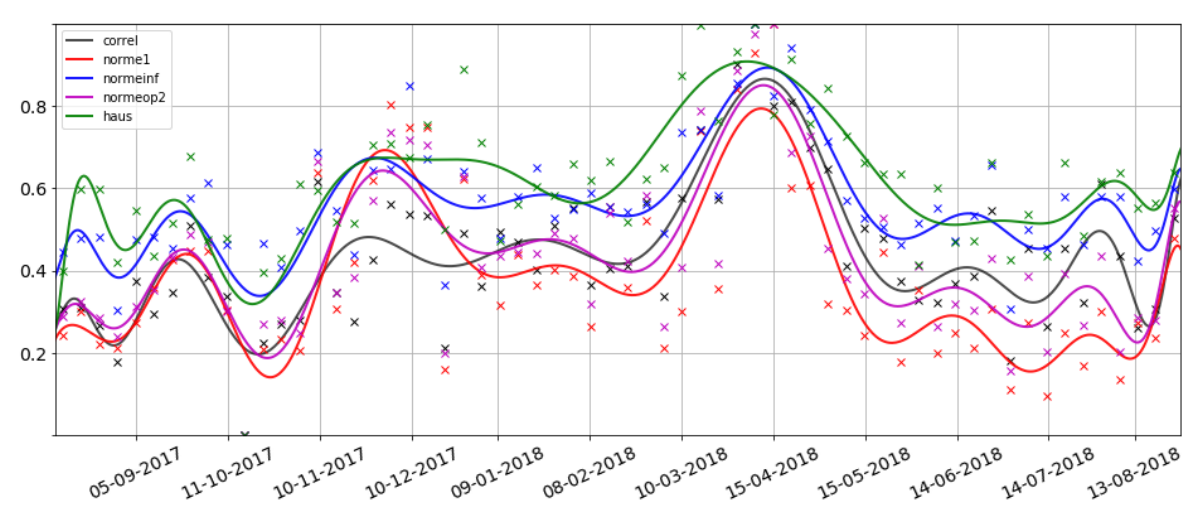

(a)

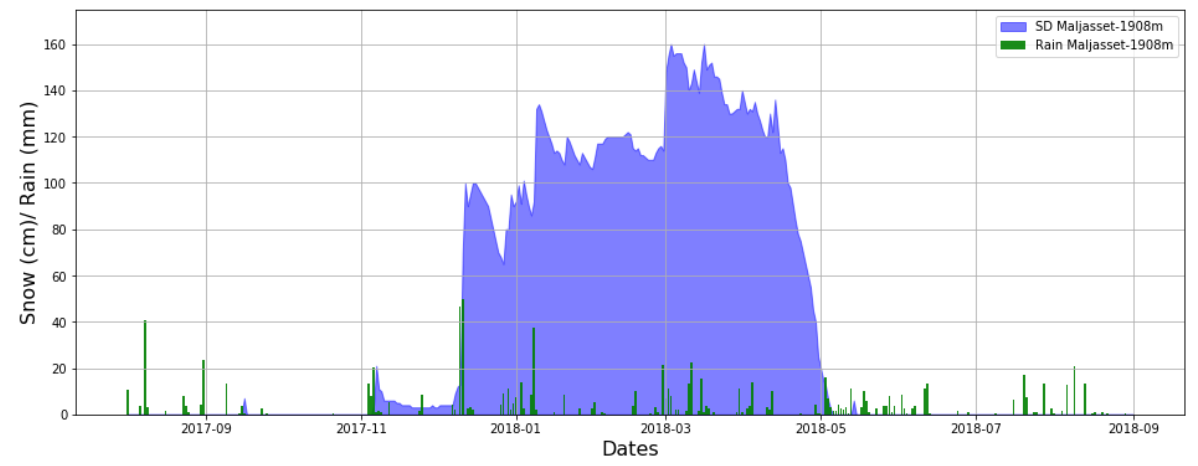

(b)

Figure 5.6: (a) Metrics computed over test area3 near Maljasset station using $17 / 10 / 2017$ image as reference. The black points correspond to the correlation (5.8) between the current image $A$ and the reference image $B$ corresponding to 17/10/2017. The red and blue points correspond to the distances (5.5) with $p=1$ (mean error) and $p=\infty$ (maximal error), respectively, and the magenta points to the distance 5.6 derived from the spectral norm. The green points corresponds to the Hausdorff distance (5.7). Each distance is rescaled by its maximal value over the time series. The curves correspond to least square fits using Legendre polynomials. (b) Snow depth and daily rain rates observed at Maljasset station.

distances). When using October 17 th as reference, the maximal error $\mathrm{d}_{\infty}$ and Hausdorff distance $\mathrm{H}_{\log }$ (blue and green curves) vary similarly, but the computation of the maximal error is much faster. We also observe similar variations between the mean error $\mathrm{d}_{1}$ and spectral norm $\widetilde{\mathrm{d}}_{2}$ (red and magenta curves). Almost all distances display a peak for the melting period indicating a strong dissimilarity with the chosen 


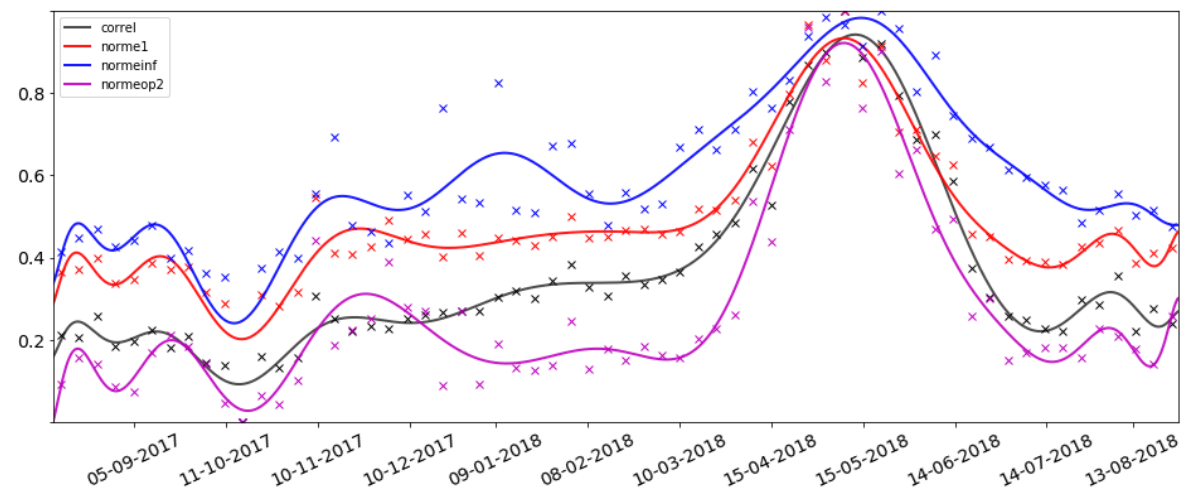

(a)

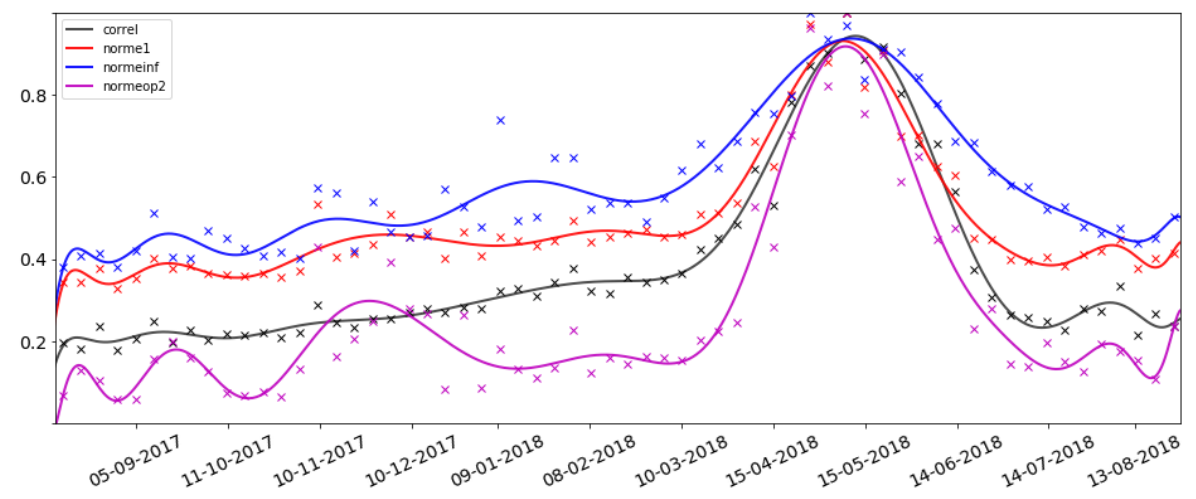

(b)

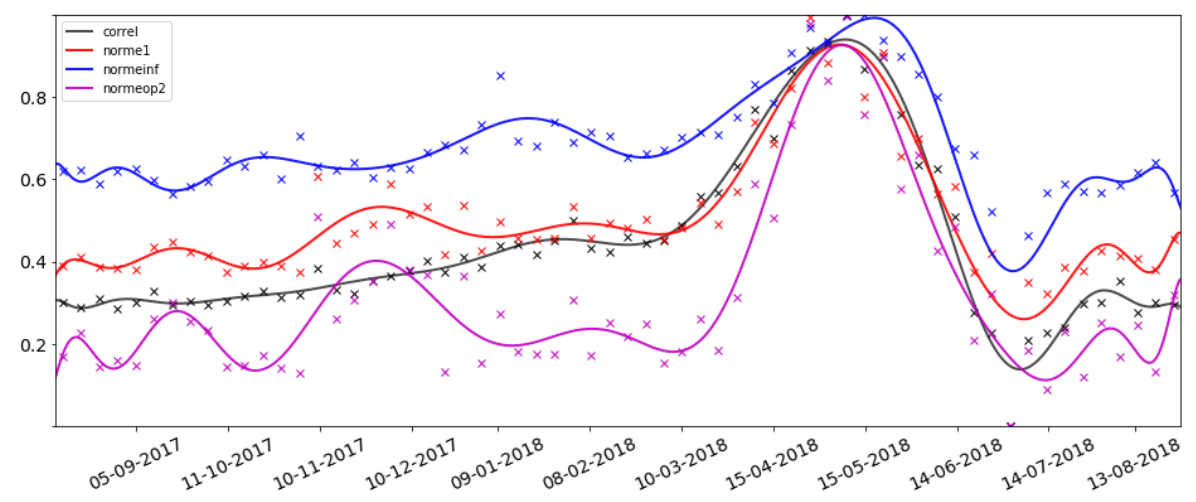

(c)

Figure 5.7: (a) Same as Figure 5.6 a) but for test area2 using 17/10/2017 as reference, (b) same as (a) but using 06/08/2017 as reference image (c) same as (a) but using $02 / 07 / 2018$ as reference image 


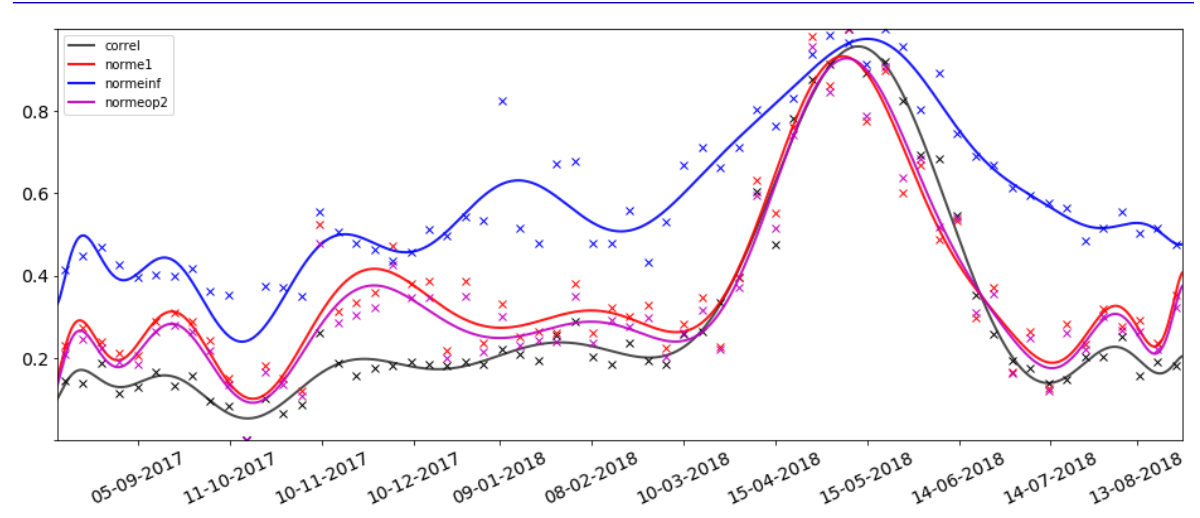

(a)

Figure 5.8: Same as Figure 5.7 (a) but using pixels for which the logarithm of the ratio between the current image $A$ and the reference $B(17 / 10 / 2017)$ is negative. Black points : correlation (5.12). Red and blue : distances (5.9) with $p=1$ (mean error) and $p=\infty$ (maximal error). Magenta : distance [5.10] derived from the spectral norm. Each distance is rescaled by its maximal value over the time series and the curves correspond to least square fits with Legendre polynomials.

reference (slightly earlier using the Hausdorff distance). A secondary distance peak is detected (mainly using $\mathrm{d}_{1}$ and $\widetilde{\mathrm{d}}_{2}$ ); this peak would correspond to the presence of wet snow following the first snowfall in early November on a still warm soil. Fluctuations in the distances are observed for some events often related to rain on snow.

Figure 5.7 provides a complement to these statistics by showing the distance results using test area 2 and by selecting two other reference dates: 06/08/2017 (Figure 5.7(b)) and $02 / 07 / 2018$ (Figure 5.7(c)) in addition to 17/10/2017. The main peak is detected, generally later on with the maximal error $\mathrm{d}_{\infty}$. Note that the date of the maximum amount of snow, and therefore the beginning of the melting, is not the same between area2 and area3. Globally, the distributions of the distances according to the selected reference are quite similar with an overall less flat distribution for the spectral norm $\widetilde{\mathrm{d}}_{2}$. For the larger area2, $\widetilde{\mathrm{d}}_{2}$ and $\mathrm{d}_{1}$ display significantly different time series, unlike for the small area3. The Hausdorff distance is omitted because of its time-consuming computation in this larger area.

In Figure 5.8, we restrict the computation of Figure 5.7 (a) to pixels for which the logarithm of the ratio between the current image A and the reference B (17/10/2017) is negative. This is done by replacing the similarity measures $(5.5)-(5.8)$ by the modified ones 5.9-5.12. With this pixel selection, the mean error $\mathrm{d}_{1}$ and spectral norm $\widetilde{\mathrm{d}}_{2}$ 


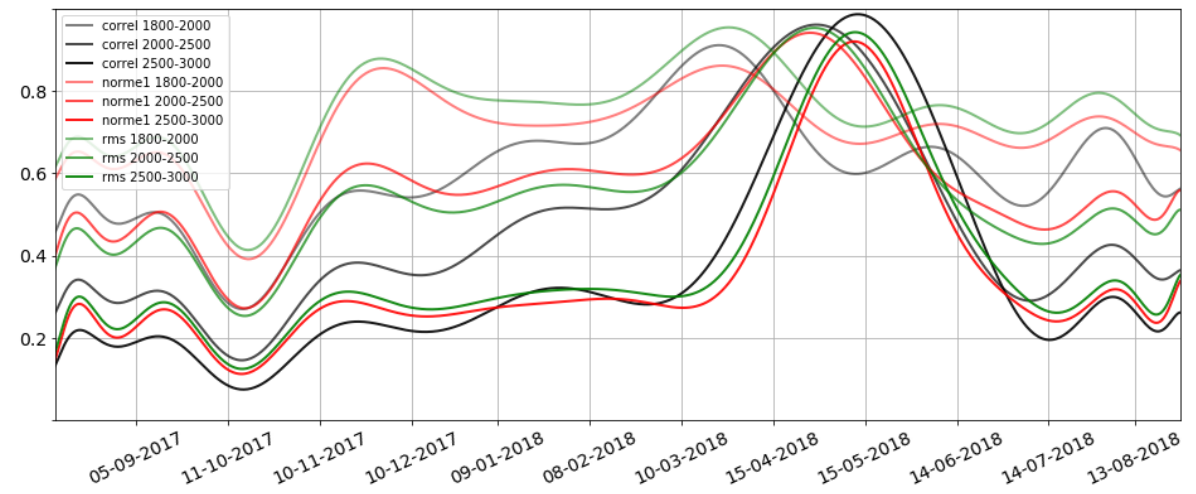

Figure 5.9: Selection of metrics computed over test area2 for ranges of altitudes: $1800-2000 \mathrm{~m}, 2000-2500 \mathrm{~m}$ and $2500-3000 \mathrm{~m}$; the reference image is $17 / 10 / 2017$. We consider the correlation (5.8) (black curves), the mean error (5.5) with $p=1$ (red curves) and the rms error (5.5) with $p=2$ (green curves). The distances are modified according to formula (5.13)-(5.14), where $\Gamma$ denotes the subset of pixels whose altitudes belong to the given range. Each distance is rescaled by its maximal value over the time series and the curves correspond to least square fits with Legendre polynomials.

(red and magenta curves) display very similar variations, while the computation of $d_{1}$ is numerically less expensive.

The timing of the peak of the maximum distance is variable depending on the altitude. Figure 5.9 shows the distances by altitude intervals $(1800-2000 \mathrm{~m}, 2000$ $2500 \mathrm{~m}, 2500-3000 \mathrm{~m}$ ) and highlights that this peak is delayed for high altitudes. The distribution of distances is also less flat for high altitudes, with the exception of the distance increase in early November at $1800-2000 \mathrm{~m}$, which is detected mainly using the mean error $\mathrm{d}_{1}$ and $\mathrm{rms}$ error $\widetilde{\mathrm{d}}_{2}$. One can notice that these two distances display similar variations, but the numerical evaluation of the rms error is more expensive.

\subsection{Conclusion}

In this chapter, we report on results from a first evaluation study of the potential of different mathematical metrics that would help to better detect wet snow pixels in SAR image time series. Particular emphasis was placed on methods that would allow optimal selection of the reference images that are required to apply change detection methods. The correlation values of the images over the whole time series offer some very promising possibilities for the selection of reference images. The criteria for such a selection still remain to be studied in depth. The different metrics 
THRESHOLDS AND DISTANCES TO BETTER DETECT WET SNOW OVER MOUNTAINS WITH SENTINEL-1 IMAGE TIME SERIES 17

studied show variations that can be linked to significant changes in the SAR signal (related to rain-on-snow episodes, snow humidification, ...) which is promising for implementing inversion methods integrating the time series of images as a whole. The distances introduced in this work could be improved in several ways :

- Filtering effects (Starovoitov \& Samal (1998)) could be introduced in a nontrivial way, taking into account the terrain characteristics such as altitude, orientation and slope.

- Given the relatively larger size of full images (test area1 of the order of $11000 \times 11000$ pixels), it will be necessary to achieve a good balance between the computational cost of the distance functions and their efficiency for wet snow detection.

\subsection{Bibliography}

Baghdadi, N., C. L. \& Bernier, M. (1998), 'Airborne c-band sar measurements of wet snow-covered areas', IEEE Transactions on Geoscience and Remote Sensing 36(6), 1977-1981.

Baghdadi, N., Gauthier, Y., Bernier, M. \& Fortin, J.-P. (2000), 'Potential and limitations of radarsat sar data for wet snow monitoring', IEEE T. Geosci Remote 38(1), 316-320.

Di Gesu, V. \& Starovoitov, V. (1999), 'Distance-based functions for image comparison', Pattern Recognition Letters 20, 207-214.

Gascoin, S., Grizonnet, M., Bouchet, M., Salgues, G. \& Hagolle, O. (n.d.), 'Theia snow collection: high-resolution operational snow cover maps from sentinel-2 and landsat-8 data,', Earth Syst. Sci. Data 11, 492-514.

Goetz, D. (2018), 'Bilan nivo-météorologique de l'hiver 2017-2018', Revue de l'ANENA https://www.anena.org/5042-la-revue-n-a.htm.

Karbou, F., Veyssière, G., Coléou, C., Dufour, A., Gouttevin, I., Durand, P., Gascoin, S. \& Grizonnet, M. (2021), 'Monitoring wet snow over an alpine region using sentinel-1 observations', IEEE Trans. Geoscience and Remote Sensing 13(3), 381.

Koskinen, J., Pulliainen, J. \& Hallikainen, M. (1997), 'The use of ers-1 sar data in snow melt monitoring', IEEE Transactions on Geoscience and Remote Sensing 35, 601-610.

Luojus, K., Pulliainen, J., Metsamaki, S. \& Hallikainen, M. (2007), 'Snow-covered area estimation using satellite radar wide-swath images', IEEE Trans. Geosci. Remote Sens. 45, 978-989.

Magagi, R. \& Bernier, M. (2003), 'Optimal conditions for wet snow detection using radarsat sar data', Remote Sens. Environ. 84, 221-233.

Marin, C., Bertoldi, G., Premier, V., Callegari, M., Brida, C., Hürkamp, K., Tschiersch, J., Zebisch, M. \& Notarnicola, C. (2020), 'Use of sentinel-1 radar 
observations to evaluate snowmelt dynamics in alpine regions', The Cryosphere 14, 935-956.

Nagler, T. \& Rott, H. (2000), 'Retrieval of wet snow by means of multitemporal sar data', IEEE Transactions on Geoscience and Remote Sensing 38, 754 - 765.

Nagler, T., Rott, H., Ripper, E., Bippus, G. \& Hetzenecker, M. (2016), 'Advancements for snowmelt monitoring by means of sentinel-1 sar', Remote Sensing 8(4).

URL: http://www.mdpi.com/2072-4292/8/4/348

Starovoitov, V. \& Samal, D. (1998), 'Experimental study of color image similarity', Machine Graphics and Vision 11, 455-462.

Stoffel, M. \& Corona, C. (2018), 'Future winters glimpsed in the alps', Nature Geoscience 11, 458-460.

Tsai, Y. L., Dietz, S., Oppelt, A. \& Kuenzer, N. (2019), 'Wet and dry snow detection using sentinel-1 sar data for mountainous areas with a machine learning technique', Remote Sensing 11, 895.

Zamperoni, P. \& Starovoitov, V. (1996), 'On measures of dissimilarity between arbitrary gray-scale images', International Journal of Shape Modeling 2, 189-213. 